\title{
Sarilumab for the treatment of rheumatoid arthritis
}

\section{Simon Cooper is interviewed by Ellen Clarke, Commissioning Editor}

Simon Cooper has $>18$ years of global experience in the pharmaceutical industry. He joined Sanofi in July 2014 as the Vice President, Global Project Head. In his current position at Sanofi, Dr Cooper is responsible for the clinical development of sarilumab and the worldwide submission in rheumatoid arthritis. He joined Sanofi after serving as the Global Program Medical Director at Novartis since 2012. In this role, Dr Cooper acted as the clinical lead for secukinumab psoriasis submission. Prior to Novartis, Dr Cooper held various posts at Human Genome Sciences, USA, including Executive Director of Clinical Research, Senior Director of Clinical Research and Director of Clinical Research. During his tenure at Human Genome Sciences, USA, Dr Cooper was involved in the submission of belimumab leading to its approval for SLE, and was responsible for its subsequent clinical development program. Dr Cooper has also previously held positions at MedImmune Ltd, UK, Roche, Napp Pharmaceutical Research Ltd, Wyeth Research and Medeval Ltd. In these roles, his responsibilities ranged from medical oversight of clinical trials to medical support for commercial, medical affairs and business development. He received a Bachelor of Medicine and Bachelor of Surgery from University of Newcastle upon Tyne Medical School.

First draft submitted: 18 November 2015; Accepted for publication: 18 December 2015; Published online: 10 February 2016

Keywords: anti-TNFs •IL6 inhibitor $\bullet$ Regeneron $\bullet$ rheumatoid arthritis $\bullet$ Sanofi $\bullet$ sarilumab - tocilizumab

Q What are the current challenges associated with the treatment of rheumatoid arthritis?

We have seen significant improvements in our ability to treat rheumatoid arthritis patients over the last few years. I think what is clear is that not all drugs work on all patients, which makes new treatment options essential.

Q Can you give us a bit of background behind the sarilumab TARGET study?

The most common biological treatments used in rheumatoid arthritis are ones that target the cytokine TNF. The TARGET study was designed to evaluate the efficacy and safety of sarilumab in patients with active rheumatoid arthritis who had an inadequate response or were intolerant to these anti-TNF therapies. We found that sarilumab was highly effective in difficult to treat patients, with a response consistent with what we had seen in the MOBILITY trial, the trial involving patients who were new to biological therapies. TARGET was the second of our pivotal Phase III program. As previously mentioned, we had MOBILITY, which was a study of rheumatoid arthritis patients who had an inadequate response or who were intolerant to methotrexate, and that also included radiographs. Patients had $\mathrm{X}$-rays at various time points to measure the actual impact to the bone. As well as
Simon Cooper

Vice President, Global Project Head, Immunology \& Inflammation, Sanofi, USA

Simon.cooper@sanofi.com 
TARGET, which is the TNF-inadequate responder, we carried out a study in monotherapy patients called SARIL-RA. We also had a study called ASCERTAIN that was a safety calibrator study against tocilizumab, and had a study called EASY that was looking at a device for patients.

Q Can you explain how the sarilumab treated patients compared with the placebo groups in the TARGET study?

In TARGET, we had two coprimary endpoints. One looking at the improvement in overall disease activity, called the ACR20 response, and the other one looking at patient function, called the HAQ-DI. We saw improvements in signs and symptoms as measured by the ACR20 at week 24. The proportion of patients achieving an ACR 20 response was $61 \%$ in the sarilumab $200 \mathrm{mg}$ group, $56 \%$ in the sarilumab $150 \mathrm{mg}$ group and $34 \%$ in the placebo group. We saw statistically significant differences between both sarilumab treatment groups compared with placebo. For HAQ-DI, which assesses the patient's ability to perform daily physical activities, we measured at baseline and at week 12 . We saw changes from baseline in HAQ-DI of $-0.49,-0.50$ and -0.29 in the sarilumab $200 \mathrm{mg}$, sarilumab $150 \mathrm{mg}$ and placebo groups at week 12. Again, we saw statistically significant differences for both of the sarilumab groups.

Q Can you tell us about the mechanisms of action of sarilumab?

Sarilumab targets the IL-6 receptor. We know that IL-6 is a key cytokine in the development of rheumatoid arthritis and it is implicated not only in the joint inflammation that we see, but also the systemic symptoms that patients experience such as fatigue and anemia.

Q Were there any adverse events recorded in the TARGET study?

Treatment emergent adverse events were more frequently recorded in the sarilumab groups. We recorded $65 \%$ in the $200 \mathrm{mg}$ sarilumab group and $66 \%$ in the $150 \mathrm{mg}$ group versus $50 \%$ in the placebo group. There was a higher incidence of serious adverse events in the $200 \mathrm{mg}$ sarilumab group compared with the placebo group: 5\% for $200 \mathrm{mg}$ sarilumab versus 3\% for placebo, but the event rate for $150 \mathrm{mg}$ was also $3 \%$. The most frequently reported adverse events were infections. We saw $30 \%$ in $200 \mathrm{mg}$ sarilumab, $22 \%$ in $150 \mathrm{mg}$ and $27 \%$ in the placebo group. Serious infec- tions were very low and occurred in two patients in the $200 \mathrm{mg}$ sarilumab group, one in the $150 \mathrm{mg}$ group and two in the placebo group.

\section{Q Are there any follow-ups on the studies planned?}

We have an ongoing trial at the moment, which is a study of sarilumab monotherapy head-to-head against monotherapy with an anti-TNF agent. We are also discussing additional studies we would like to do with sarilumab, but it is early days at the moment.

\section{Q How does sarilumab compare with other IL-6} inhibitors?

We do not have direct head-to-head efficacy data against the current IL-6 inhibitor tocilizumab. We did, however, conduct a study called ASCERTAIN, which was a safety calibrator study, and that included tocilizumab. What we can say is that the safety of these two molecules appears broadly comparable.

\section{Q Why is immunotherapy an attractive} treatment strategy for rheumatoid arthritis?

As our understanding of the disease has progressed, immunotherapies present an opportunity for us to be more targeted in our approach to treatment. For example, with sarilumab, we target the IL-6 receptor, and we know that IL-6 is a key cytokine that is implicated not only in the inflammatory processes that we see in the joints but also in many of the systemic features that these patients experience. Although we have become better at treating these patients in the years that we have been developing drugs for them, it is clear that there are still a number of patients who do not respond to therapies, so having new options available for them is tremendously important.

Financial \& competing interests disclosure

$S$ Cooper is employed at Sanofi and may have stock or stock options. The authors have no other relevant affiliationsor financial involvement with any organization or entity with a financial interest in or financial conflict with the subject matter or materials discussed in the manuscript apart from those disclosed.

No writing assistance was utilized in the production of this manuscript.

\section{Disclaimer}

The opinions expressed in this interview are those of the interviewees and do not necessarily reflect the views of Future Medicine Ltd. 